Beins, Hans Jürgen; Lensing-Conrady, Rudolf; Wolf, Guido: Von Sinnen. Impulse und Interventionen für Meetings, Workshops, Konferenzen. Ein Methodenbuch. Verlag modernes lernen, Dortmund, 2017, 144 Seiten, $€$ 18,80 (D)

Erlebnisreiche Events für Mitarbeiterlnnen sowie Spielgelegenheiten direkt am Arbeitsplatz gewinnen nicht nur in den Kreativabteilungen von Unternehmen, sondern auch in Form von Teambuilding-Aktionen und Bewegungspausen in Meetings von kleinen und großen Teams an Bedeutung. Ihnen werden eine erholsame Aktivierung zugesprochen sowie die Förderung von Motivation, Kreativität und insbesondere von Problemlösefähigkeit - einer Metakompetenz, die für individuelle und teamorientierte Arbeitsprozesse besonders wichtig erscheint. Mit $\gg$ Von Sinnen « legt das Autorenteam Hans Jürgen Beins, Rudolf Lensing-Conrady und Guido Wolf ein Methodenbuch für eben solche Aktionen vor.

In einer kurzen Einleitung wird der Zusammenhang von Organisationsentwicklung und der damit verbundenen Sinn-Suche mit der sinnlichen Wahrnehmung und ihrer Förderung hergestellt, wodurch sich die Titelauswahl erschließen lässt. Die Autoren schildern ihre Erfahrung als Begleitervon Entwicklungsprozessen im Kindes-, Jugendund Erwachsenenalter und als Leiter von Fortbildungs- und Beratungsangeboten sowie ihre Motivation, das vielfach erprobte Repertoire zusammenzutragen und für andere Trainerlnnen, Coaches, ModeratorInnen oder Facilia- tor zur Verfügung zu stellen. Eine kurze Gebrauchsanweisung leitet über in den Hauptteil mit einer umfangreichen und bebilderten Zusammenstellung von Spielen, Übungen, Impulsen und Interventionen, deren Begriffe von den Autoren synonym verwendet werden. Nach Materialeinsatz geordnet werden in drei Kapiteln insgesamt 51 bewegungsorientierte Ideen vorgestellt: Mit vollem Einsatz - ohne Material; mit Alltagsmaterialien (z.B. Zollstöcke, Zeitungen und Teppichfliesen) sowie MiniMat-Minimaler Materialaufwand (z. B. Moderationskarten, Stifte, Klebeband und Luftballons). Jede Übung wird auf einer Doppelseite zunächst zusammengefasst nach: Teilnehmerzahl, Organisationsform, Zeitrahmen, Ort, Material, Schwerpunkt, Charakteristik, Positionierung. Die LeserInnen können sich somit einen kurzen Überblick verschaffen und erhalten neben einem Foto der Aktion eine detaillierte Beschreibung sowie Hinweise zum Ziel und zu den Effekten der Übung für die Teilnehmenden. Als solche werden beschrieben: spielerisch in Bewegung kommen, lockere Form der Begegnung, ungewöhnliche Perspektiven einnehmen, das Denken "out of the box", Risikobereitschaft erfahren, usw.

In einem Nachwort geben die Autoren einen Überblick über die

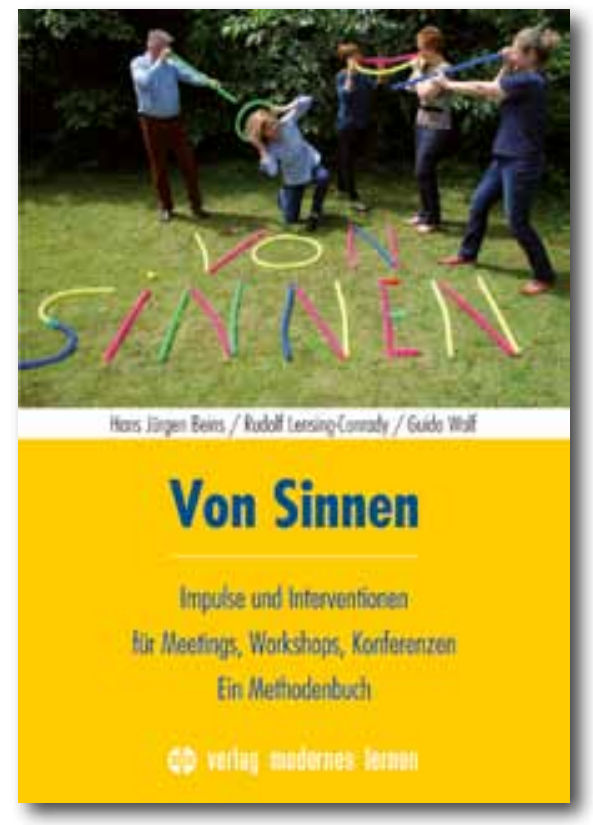

Wahrnehmungssysteme und weisen anschließend auf Literatur und Adressen zur Materialbestellung sowie Workshops hin.

Die hier zusammengestellten Interventionen wirken abwechslungsreich und sprechen die Experimentierfreude, eine lockere Kontaktaufnahme und das gemeinsame Lösen von ungewöhnlichen Aufgaben in der Gruppe an. Nicht alle Inhalte sind neu, stehen hier jedoch in einem neuen Zusammenhang und verfolgen eine klare Zielsetzung für Fachkräfte, die miteinander arbeiten. Die gut durchdachte Struktur, die Anordnung und farbige Illustration tragen im hohen Maße zur Orientierung 
und zum Verständnis bei. Insbesondere der Einsatz einfacher Materialien und die Entwicklung individueller Variationen könnte die angesprochene Zielgruppe der Beraterlnnen, Coaches und Moderatorlnnen überzeugen, einige Impulse in der Praxis umzusetzen und somit einen echten Mehrwert für ihre Arbeit mit Gruppen im beruflichen Kontext bieten. Die Ästhetik und Symbolik des Titelfotos weisen allerdings - im Gegensatz zu den Fotos im
Buch - auf Sehgewohnheiten im Zusammenhang mit bunten Kinderspielen und Freizeitangeboten hin und werden den Inhalten und dem Nutzen für professionelles Handeln nicht gerecht. Ein fokussierteres Titelbild hätte den Zugang für Fachkräfte auch außerhalb der Psychomotorik-Landschaft vermutlich vereinfacht.

Seminarleitungen und Trainerlnnen können ein sinnvoll aufbereitetes und gut verständliches Methoden- buch erwarten, das mit einfachen oder komplexeren Interventionen - und in Kombination mit einer behutsamen, prozessorientierten Herangehensweise - mehr Bewegung in die Begleitung von Gruppen- und Arbeitsprozessen bringen kann.

\section{Caterina Schäfer}

DOI 10.2378/ motorik2018.artogd 